\title{
Challenges in Medical Journalism: Reflections from the Year Past!
}

\author{
Saba Sohail \\ Department of Radiology, Dow University of Health Sciences and Civil Hospital, Karachi, Pakistan
}

While ensuring the publication of high-quality manuscripts is the editors' task, its execution has always been challenging. ${ }^{1}$ Some of these are perennial issues like ethical committee approval, duplication / redundant publications, covert or undeclared pharma-industry support, authors' non-compliance with the requirements and, therefore, increasing desk rejection rate without peer review, etc. Some issues are new and mostly arisen out of technical advances, which have been boon and bane at the same time, including predatory publishers and publications, the various open access models and their advantages and disadvantages, increasing submissions from across the Globe with associated issues. These challenges, which affected the Journal of College of Physicians and Surgeons Pakistan (JCPSP), have been felt and discussed in international forums such as World Association of Medical Editors (WAME), and Pakistan Association of Medical Editors (PAME). The present write-up is a reflection based upon these challenges. A brief outline is also given how these were addressed. This may be of help to other editors who may be facing similar dilemmas.

The menace of predatory publications has long since made its presence felt; and awareness has been created and extended to fight against it. ${ }^{2}$ Another problem is managing the citation of the predatory publications by the uncanny researchers. Tracing the research that is authentic but published in a predatory journal, and differentiating it from a completely bogus publication in a similar journal is a herculean task, indeed. On the other hand, misleading reports still find their way to publishing in authentic and respectable journals too, e.g., the 2016 Excel Trial.

The urge for visibility and access to a wide range of authors and readers in order to achieve more citation led to the development of various open access models. Here, it is usually the author who bears the expenses of publication in the form of article processing and publication charges. While waivers and discounts exist in some cases and conditions, a vast majority of these open-access journals remain out of pay-limits for the resource-limited authors. The authors, who pay for

Correspondence to: Prof. Saba Sohail, Department of Radiology,

Dow University of Health Sciences and Civil Hospital,

Baba-e-Urdu Road, Karachi, Pakistan

E-mail:drsabasohail@hotmail.com

Received: January 03, 2020; Revised: January 06, 2020;

Accepted: January 06, 2020 publishing, accordingly raise their own rather justified demands. The loudest of these is the need to have crystal-clear, explicit, and detailed instructions to authors regarding the format requirements. These differ from journal to journal according to the house-style so that there is considerable signature variety differentiating the style of different journals in format, word counts, referencing, and citation which frustrate authors and editors alike when they write in, and receive an unfamiliar format, respectively. Even the style of reference citations confuses particularly the junior researcher when asked to switch between numbering and naming styles. Despite the availability of various reference-rectifying software, which may be used by both the authors and the publishers, there always remain subtle details that have to be manually rectified. Hence, there is an increasing demand from authors that all research journals follow a similar pattern of archival format. The ease of trans-border trans-lingual submissions with increasing submissions from non-native writers has compounded this issue.

The "uniform requirements" are recommendations for all practical purposes. To overcome these variations and archival deficiencies in submissions, many journals advise authors to use professional editing services. While this is often adopted by authors from non-English speaking countries, these services can translate or paraphrase for routine English usage and correct syntax errors; translations of technical jargons remain compromised. Many journals including JCPSP carry out substantive comprehensive editing in such cases after acceptance of articles whose science is better than the syntax, but this can become exhausting when faced with a large number of write-ups that editors are assigned to comprehensively rectify to fit in the journal format. This can lead to editor burn out, too. Another mercenary aspect arising out of this is forcing the authors to use a particular editing service. This also suggests a predatory intent or link. In fact, a recent study estimated the financial impact of such formatting requirements and time spent thereon to be a staggering 1.1 billion US dollars. ${ }^{3}$

Other challenges are the peer review process, sharing reviewers' lists with indexing agencies, and the breach of confidentiality in doing so. While many journals reward their reviewers monetarily; and majority acknowledge them by printing a general list of reviewers in a specified issue; usually the last volume of the year, sharing the details of reviewers by any agency is considered unethical. 
A published research is the only viable record that a research was conducted and what it led to. Evidencebased medicine is nothing but data and practices derived from such records. It is, therefore, imperative that this published permanent record of evidence should conform to established ethical practices and be authentic. Editors are not policemen; they can only provide guidance and awareness against the challenges and threats that may affect the process of collecting sound, correct and useful evidence. There is a dire need for developing concerted efforts to face the ethical and technical threats to the profession. The most effective way is to develop platforms for sharing these experiences; and how the established, most respected editors and publishing houses, dealt with these upcoming challenges.

WAME, Eastern Mediterranean Association of Medical Editors (EMAME), European Association of Science Editors (EASE), and Pakistan Association of Medical Editors
(PAME) in Pakistani context, remain very useful platforms in meeting such challenges, providing guidelines, and sharing experiences.

\section{REFERENCES}

1. Galipeau J, Cobey KD, Barbour V, Baskin P, Bell-Syer S, Deeks J, et al. An international survey and modified Delphi process revealed editors' perceptions, training needs, and ratings of competency-related statements for the development of core competencies for scientific editors of biomedical journals. F1000Res 2017; 6:1634.

2. Cukier S, Helal L, Rice DB, Pupkaite J, Ahmedzai N, Wilson M, et al. Checklists to detect potential predatory biomedical journals: A systematic review, available at www.medrxiv.org/ content/10.1101/19005728v1.

3. Jiang Y, Lerrigo R, Ullah A, Alagappan M, Asch SM, Goodman SN, et al. The high resource impact of reformatting requirements for scientific papers. PLoS One 2019; 14:e0223976. 